\title{
Determinants of Under-five Deaths in Urban Parts of Ethiopia
}

\author{
Yenew Alemu ${ }^{1}$, Mekonnen Tadesse ${ }^{2}$ \\ ${ }^{1}$ Department of Statistics, College of Natural and Computational Science, Injibara University, Injibara, Ethiopia \\ ${ }^{2}$ Department of Statistics, College of Natural and Computational Science, Addis Ababa University, Addis Ababa, Ethiopia
}

\section{Email address:}

yenewalemu@gmail.com (Y.Alemu)

\section{To cite this article:}

Yenew Alemu, Mekonnen Tadesse. Determinants of Under-five Deaths in Urban Parts of Ethiopia. Science Journal of Applied Mathematics and Statistics. Vol. 8, No. 4, 2020, pp. 53-59. doi: 10.11648/j.sjams.20200804.12

Received: July 28, 2019; Accepted: September 27, 2019; Published: September 21, 2020

\begin{abstract}
Introduction: Under-five mortality rate is defined as the probability (expressed as a rate per 1000 live births) of a child born in a specified year dying before reaching the age of five years subject to current age-specific mortality rates (UNICEF 2008). Objects: The objective of this study was to identify determinants of under-five mortality in urban parts of Ethiopia using the 2011 EDHS data. Methods: To achieve the objective of this study descriptive statistics and count regression models were used for data analysis using socio-economic, demographic and environmental related variables as explanatory variables and the number of under-five deaths per mother as the response variables. Results: Information on the number of deaths of under-five children obtained from a total of 1,807 women in the urban parts of Ethiopia was studied. From these, 328 women experienced 513 under-five deaths. From these, 328 women experienced 513 under-five deaths. Conversely, $81.8 \%$ of the women in urban areas never experienced under-5 death of their children. Nevertheless, $12 \%, 3.6 \%, 1.6 \%, 0.6 \%, 0.2 \%$, $0.1 \%$ and $0.1 \%$ of them lost $1,2,3,4,5,6$ and 7 of their under-five deaths, respectively. Conclusion: Factors influencing the number of under-five deaths have been identified. Mother's level of education, age of mothers at the first birth, toilet facility and work/employment status of mothers were found to be statistically significant with the number of under-five deaths per mothers in urban parts of Ethiopia.
\end{abstract}

Keyword: Urban, Under Five Mortality, Count Regression Model

\section{Introduction}

Under-five mortality rate is defined as the probability (expressed as a rate per 1000 live births) of a child born in a specified year dying before reaching the age of five years subject to current age-specific mortality rates [1]. It has several advantages as a barometer of child wellbeing in general and child health in particular. First, it measures an outcome of the development process rather than an input, such as per capita calorie availability or the number of doctors per 1,000 population - all of which are means to an end. Second, the U5MR is known to be the result of a wide variety of inputs: the nutritional status and the health knowledge of mothers; the level of immunization and oral rehydration therapy; the availability of maternal and child health services (including prenatal care); income and food availability in the family; the availability of safe drinking water and basic sanitation; and the overall safety of the child's environment, among other factors. Third, the U5MR is less susceptible to the fallacy of the average than, for example, per capita gross national income (GNI per capita). This is because the natural scale does not allow the children of the rich to be 1,000 times as likely to survive, even if the human-made scale does permit them to have 1,000 times as much income. In other words, it is much more difficult for a wealthy minority to affect a nation's U5MR, and it therefore presents a more accurate, if far from perfect, picture of the health status of the majority of children (and of society as a whole).

Despite the fact that a number of researchers have studied the risk factors of under-five mortality in Ethiopia, the majority of them studied the risk factors at national level. Such studies have overlooked an important point for policy makers as the findings at national level may not demonstrate the exact situation at urban areas. Under-five death is 
showing a declining trend over the last decades. According to [2], report the level of under-five mortality in urban area is 83 deaths per 1000 live births.

Logistic regression cannot provide sufficient information for studying the pattern of multiple child deaths. Therefore, we can develop and compare count regression models for the number of under-five deaths and discuss how they can enhance our understanding of the risk factors of under-five deaths. The general objective of this study is to identify the risk factors of under-five mortality in Urban Ethiopia.

\section{Methods}

\subsection{Source of Data}

The source of the data in this study is the 2011 Ethiopia Demographic and Health Survey (EDHS). SPSS version 23 and STATA version 14 were used as tools of analysis.

\subsection{Variables in the Study}

Detail description and coding of the demographic, socioeconomic, and environmental factors to under-five mortality is presented in Tables 1, 2, and 3 respectively.

\subsection{Data Analysis}

In this study, the variable of interest is a count variable. Count data is non negative, integer, non-normal and etc. For this study four count regression models.

\subsubsection{Poisson Regression Model}

In the poisson regression model, mean $=$ variance. Let $\mathrm{Y}_{\mathrm{i}}$ represent counts of events occurring in a given time or exposure periods with rate $\mu_{\mathrm{i}} . \mathrm{Y}_{\mathrm{i}}$ are Poisson random variables which the p. m. f. is characterized by

$$
\mathrm{P}\left(\mathrm{Y}_{\mathrm{i}}=\mathrm{y}_{\mathrm{i}}, \mu\right)=\frac{\mathrm{e}^{-\mu_{\mathrm{i}} \mu_{\mathrm{i}} \mathrm{y}_{\mathrm{i}}}}{\mathrm{y}_{\mathrm{i}} !}, \mu_{\mathrm{i}}>0, \mathrm{i}=1,2, \ldots . \mathrm{n} \text { and } \mathrm{y}_{\mathrm{i}}=0,1,2, \ldots
$$

where, $y_{i}$ denotes the value of an event count outcome variable occurring in a given time or exposure periods with mean parameter $\mu_{\mathrm{i}}$.

The log-likelihood function is

$$
\ell=\log (\mathrm{L}(\beta))=\sum_{i=1}^{n}\left[y_{i} \ln \mu_{i}-\mu_{i}-\ln y_{i} !\right]
$$

There are two basic criteria commonly used to check the presence of overdispersion:

1. Deviance, $\mathrm{D}(\mathrm{y}, \widehat{\mu})$, is given by

$$
\mathrm{D}(\mathrm{y}, \hat{\mu})=2 \times \sum_{i}^{n}\left\{y_{i} \ln \left(\frac{y_{i}}{\widehat{\mu}_{i}}\right)-\left(y_{i}-\hat{\mu}_{i}\right)\right\}
$$

where, $\mathrm{y}$ is the number of events, $\mathrm{n}$ is the number of observations and $\hat{\mu}_{i}$ is the fitted Poisson mean.

2. Pearson chi-square test, $x^{2}$ is also given by

$$
x^{2}=\sum_{i=1}^{n} \frac{\left(y_{i}-\widehat{\mu}_{i}\right)^{2}}{\widehat{\mu}_{i}}
$$

Over-dispersion may be a result of higher occurrence of zero counts and subject heterogeneity. If the model fits the data, both deviance and Pearson Chi-square statistics divided by the degrees of freedom are approximately equal to one. Values greater than one indicate the variance is an over-dispersion, while values smaller than one indicate an under-dispersion.

In the general Poisson regression model, we think of $\mu_{\mathrm{i}}$ as the expected number of under five-child death from the $i^{\text {th }}$ mother and the total number children ever born from the $i^{\text {th }}$ mother is $\mathrm{N}_{\mathrm{i}}$. This means parameter will depend on the population size and the total number of children ever born from the individual mother. Thus the distribution of $\mathrm{Y}_{\mathrm{i}}$ can be written as:

$$
\mathrm{Y}_{i} \sim \operatorname{poisson}\left(\mathrm{N}_{\mathrm{i}} \mu_{\mathrm{i}}\right)
$$

where $\mathrm{N}_{i}$ is the total fertility rate of $\mathrm{i}^{\text {th }}$ mother and

$$
\mu_{i}=\exp \left(X_{i}^{T} \beta\right)
$$

The logarithm of the children ever born is introduced in the regression model as an offset variable. By including ın [children ever born] as offset in the equation, it is differentiated from other coefficients in the regression model by being carried through as a constant and forced to have a coefficient of one [3]. Thus, the GLM with an offset is given by

$$
\log \mu^{T} \beta
$$

\subsubsection{Negative Binomial Regression Model}

This model is used when count data are overdispersed (i.e when the variance exceeds the mean). The NB regression model is

$$
\mathrm{P}\left(\mathrm{y}_{\mathrm{i}} ; \mu \mathrm{i}, \alpha,\right)=\frac{\Gamma\left(\mathrm{y}_{\mathrm{i}}+1 / \alpha\right)}{\mathrm{y}_{\mathrm{i}} ! \Gamma(1 / \alpha)}\left(1+\alpha \mu_{\mathrm{i}}\right)^{\frac{-1}{\alpha}}\left(1+\frac{1}{\alpha \mu_{\mathrm{i}}}\right)^{-\mathrm{y}_{\mathrm{i}}}, \mathrm{y}_{\mathrm{i}} \geq 0 \text { and } \alpha>0
$$

where, $\alpha$ shows the level of overdispersion and $Г($.$) is the$ gamma function

\subsubsection{Zero- inflated Poisson Regression Model}

The Zero-inflated Poisson regression study the relationship between dependent and independent variable(s) when there are many zeros value in the dependent variable, where the relationship is the mixture between Poisson model and Logistic model. The ZIP regression model is [4],

$$
P\left(y_{i}\right)=\left\{\begin{array}{c}
\omega_{i}+\left(1-\omega_{i}\right) e^{-\mu_{i}}, y_{i}=0 \\
\left(1-\omega_{i}\right) \frac{e^{-\mu i} \mu_{i} y_{i}}{y_{i} !}, y_{i}=1,2, \ldots, 0 \leq \omega_{i} \leq 1
\end{array}\right.
$$

where, $\mathrm{Y}_{\mathrm{i}} \sim \mathrm{ZIP}\left(\mu_{\mathrm{i}}, \omega_{\mathrm{i}}\right)$.

\subsubsection{Zero-inflated Negative Binomial Regression model}

Zero-Inflated Negative Binomial (ZINB) regression is one of the methods used in troubleshooting overdispersion due to excessive zero values in the response variable (excess zeros). The zero inflated negative binomial regression model was 
given by

$$
\mathrm{P}\left(\mathrm{y}_{\mathrm{i}} \mid \omega, \alpha, \mu\right)=\left\{\begin{array}{c}
\omega_{\mathrm{i}}+\left(1-\omega_{\mathrm{i}}\right)\left(1+\alpha \mu_{\mathrm{i}}\right)^{\frac{-1}{\alpha}}, \mathrm{y}_{\mathrm{i}}=0 \\
\left(1-\omega_{\mathrm{i}}\right) \frac{\Gamma\left(\mathrm{y}_{\mathrm{i}}+1 / \alpha\right)}{\mathrm{y}_{\mathrm{i}} ! \Gamma(1 / \alpha)}\left(1+\alpha \mu_{\mathrm{i}}\right)^{\frac{-1}{\alpha}}\left(1+\frac{1}{\alpha \mu_{\mathrm{i}}}\right)^{-\mathrm{y}_{\mathrm{i}}}, \mathrm{y}_{\mathrm{i}}>0
\end{array}\right.
$$

where, $\mu_{\mathrm{i}}$ is the mean of the underlying negative binomial distribution, $\alpha>0$ is the over dispersion parameter and is assumed not to depend on covariates and $0 \leq \omega_{i} \leq 1$. Also the parameters $\mu_{i}$ and $\omega_{i}$ depend on vectors of covariates $x_{i}$ and $z_{i}$, respectively. Newton-Raphson iteration procedure can be used for estimating the parameter of ZINB regression models.

\subsection{Goodness of Fit Tests}

\subsubsection{Likelihood Ratio Test}

$$
\mathrm{LRT}_{\alpha}=-2\left(\mathrm{LL}_{1}-\mathrm{LL}_{2}\right)
$$

This statistic has a Chi-squared distribution with 1 degrees of freedom and LL is log-likelihood. If the statistic is greater than the critical value then, the model 2 is better than the model 1 .

\subsubsection{Vuong Test}

The Vuong test is a non-nested test that is based on a comparison of the predicted probabilities of two models that do not nest [5]. The test statistic is

$$
\mathrm{V}=\frac{\sqrt{n} \frac{\sum_{i=1}^{n} m_{i}}{n}}{\sqrt{\frac{\sum_{i=1}^{n}\left(m_{i}-\bar{m}\right)^{2}}{n}}}
$$

Where, $\bar{m}$ is mean of $\mathrm{m}_{\mathrm{i}}$ and $n$ sample size.

The hypotheses of the Vuong test are:

$\mathrm{H}_{\mathrm{o}}: \mathrm{E}\left[\mathrm{m}_{\mathrm{i}}\right]=0$

$\mathrm{H}_{1}: \mathrm{E}\left[\mathrm{m}_{\mathrm{i}}\right] \neq 0$

The null hypothesis of the test is that the two models are equivalent. If $\mathrm{V}>Z_{\alpha / 2}$, the first model is preferred., if $\mathrm{V}<$ $Z_{\alpha / 2}$, the second model is preferred. And if $|\mathrm{V}|<Z_{\alpha / 2}$, none of the models are preferred.

\subsubsection{AIC and BIC}

AIC and BIC are goodness of criteria used for model selection.

AIC $=-2 \log$ likelihood $+2 \mathrm{k}$

$\mathrm{BIC}=-2 \log$ likelihood $+\mathrm{k} \ln (\mathrm{n})$

where, $\mathrm{k}=$ number of parameters and $\mathrm{n}=$ number of observations.

\section{Results}

\subsection{Descriptive Statistics}

The distribution of socioeconomic, demographic and environmental background characteristics of mothers which affect under-five child mortality is presented in Table 4. Information on the number of deaths of under-five children obtained from a total of 1,807 women in the urban parts of Ethiopia was studied. From these, 328 women experienced
513 under-five deaths. Conversely, $81.8 \%$ of the women in urban areas never experienced under-5 death of their children. Nevertheless, $12 \%, 3.6 \%, 1.6 \%, 0.6 \%, 0.2 \%, 0.1 \%$ and $0.1 \%$ of them lost $1,2,3,4,5,6$ and 7 of their underfive deaths, respectively.

Descriptive statistics for the dependent variable used in the present study are presented in Table 5 . The 1,807 observation values obtained for each variable were used in the study. Among the children of the 1,807 women considered in the study, 513 children died before the age of five. While the smallest value for the number of under-five deaths was 0 , the highest value was 7 . Out of the 1,807 observed values used in the study, 1,479 (81.8\%) were zero.

As shown in Figure 1, the distribution of the number of under-five deaths has a rapidly decreasing tail and is highly skewed to right with excess zeros.

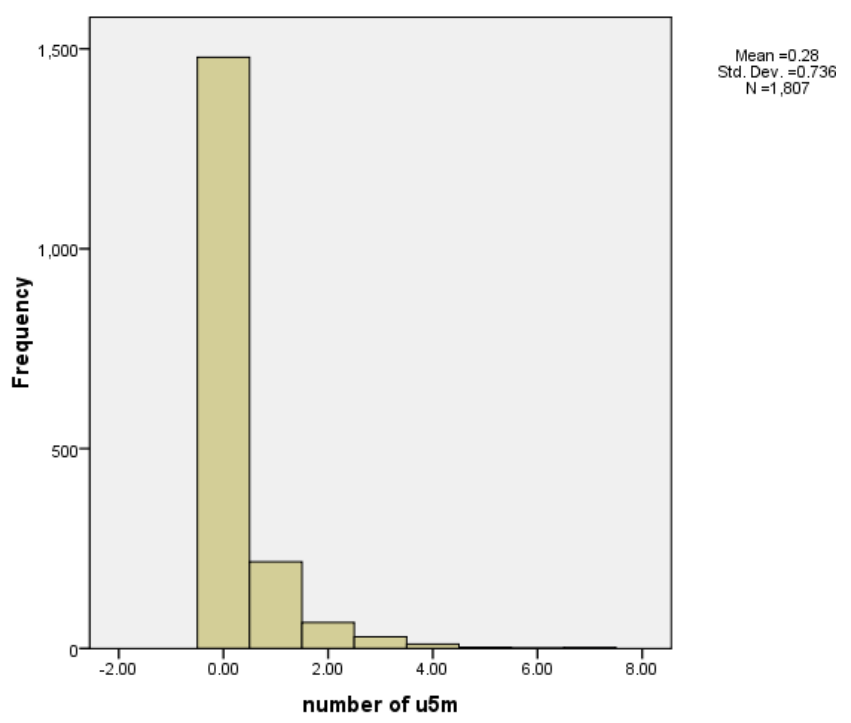

Figure 1. Histogram of the number of under-five deaths.

For urban area, both the Deviance and Pearson statistics divided by the degree of freedom is approximately one in Table 6.

The formal test for significance of over-dispersion, the likelihood ratio test of $\alpha, \mathrm{LRT}_{\alpha}=-2 \times\left(\mathrm{LL}_{\mathrm{Poisson}}-\mathrm{LL}_{\mathrm{NB}}\right)$, is computed. The likelihood ratio becomes 33.27, which corresponds to a $\mathrm{p}$-value $<0.05$, giving evidence of overdispersion. Evidence of over-dispersion indicates inadequate fit of the Poisson model. In addition, we can identify more appropriate model by using AIC, BIC and log-likelihood.

Table 7 showed that NB has maximum log-likelihood and minimum BIC and AIC indicating that the negative binomial model fits the data well. Alternatively, we can identify the non-nested models by using vuong test. The value of Vuong statistic, $\mathrm{V}=5.57$ is greater than 1.96 implying that the ZIP 
regression model is better than the Poisson regression model. In addition, $\mathrm{V}=4.54$ is greater than 1.96 implying that the ZINB model is better than the NB regression model.

As shown in the Table 9, ZIP regression model has minimum AIC and BIC and maximum log-likelihood. Moreover, the fact that the likelihood ratio of $\alpha$ statistic, $\mathrm{LRT}_{\alpha}=-2\left(\mathrm{LL}_{\mathrm{ZIP}}-\mathrm{LL}_{\mathrm{ZINB}}\right)=1.41$, is less than chi2 $(01)$, implies that the ZIP is better than the ZINB regression model since the dispersion statistic $(\alpha=0.13)$ is close to 0 .

\subsection{Interpretation of Zero Inflated Poisson Regression for Covariates of Non-zero Groups in Urban Parts of Ethiopia}

Mother's education level was an important factor for predicting the number of under-five mortality. The expected number of under-five deaths decreased by a factor of 0.50 for mothers with primary education compared to those with no education controlling other variables in the model. Also the expected number of under-five mortality for mothers with secondary and higher education was decreased by a factor of 0.19 and 0.32 as compared to those with no education, respectively, controlling other variables in the model.

As shown in Table 10, toilet facility had a significant effect on the number of under-five deaths. The expected number of under-five deaths increased by a factor 1.58 for a woman with no access to toilet facility as compared to a woman with access to toilet facility holding all other variables in the model constant.

Table 10 revealed that mother's age at the first birth has a significant effect on the number of under-five deaths. The expected number of under-five deaths for mothers in the age group 15-19 had decreased by a factor of 0.64 as compared to the expected number of under-five deaths for mothers in the age group less than 15 controlling for other variables in the model. In addition, the expected number of under-five mortality for mothers in the age group 20 and above was 50\% less than that for mothers under 15 years of age controlling for other variables in the model.

Table 10 showed that mothers work status had a significant effect on the number of under- five deaths. The expected number of under- five deaths increased by a factor of 1.59 for working mothers as compared to that for nonworking mothers while holding all other variables in the model constant.

\subsection{Interpretation of ZIP Regression for Covariate of Zero Counts in Urban Parts of Ethiopia}

Table 10 also shows that region has a significant effect on the odds of being in the always zero group. The odds of being in the zero group among children born to mothers in Harari and Addis Ababa increased by a factor of 5.86 and 3.94 to the odds among children born in Tigray, respectively, holding all other variables in the model constant.

As shown in Table 10, mother's marital status has a significant effect on the probability of being an excess zero. The odds of being in the zero groups are increased by a factor of 2.04 for unmarried mothers as compared to married mothers controlling for other variables in the model.

\section{Discussion}

This study was carried out to identify the risk factors of under-five mortality in urban areas based on EDHS 2011 data. This study has also identified the risk factors of underfive mortality in urban Ethiopia based on EDHS 2011 data. The total number of urban women covered in the present study was 1,807 of which $18.2 \%$ of them experienced underfive deaths due to different factors.

Children from urban areas, whose mothers are in a relatively older age group, were better in their survival status as compared to children whose mothers are in the younger age group. This study revealed that children born to very young (age less than 15 years) mothers are more likely to die. This finding is consistent with the findings of [6-8].

Level of education of Mothers in urban areas showed a significant effect on infant and child mortality. Under-five mortality decreased with increased level of mother's education. This result is consistent with the findings of [6, 8-9].

The under-five mortality risk was lower for women with access to toilet facility than that for women without toilet facility. This finding is consistent with $[6,10]$. In addition, children born from working mothers have higher risk of mortality than non-working mothers.

The results of ZIP indicated that region and marital status have a significant factors on the odds of being in the always zero groups. The infant and child mortality widely varied between regions in Ethiopia. The expected number of underfive deaths for women from Oromiya, Benshangul-Gumuz, SNNP and Gambela were higher than the expected number of under-five deaths for women in Tigray. But, the expected number of under-five mortality in Addis Ababa was lower than that of Tigray. This finding is confirmed by the previous researches [11-13].

\section{Conclusions}

Among the four models considered for analyzing the data from women in urban parts of Ethiopia, the ZIP regression model was found to be the most appropriate model for the data from women in urban areas. Mother's level of education, age of mothers at the first birth, toilet facility and work/employment status of mothers were found to be statistically significant with the number of under-five deaths per mother in urban parts of Ethiopia.

\section{Recommendations}

Based on this findings we recommend that there is a need for comprehensive prevention strategies that will help to further reduce child mortality, the government/ministry of health should give greater attention to improve immunization services and concentrate on health education campaigns for mothers and for the community and Early marriages should 
be discouraged and awareness about the danger of giving birth at early ages should be created through education.

\section{Author's Contributions}

Conceptualized, designed, analyzed, interpreted the results and wrote the manuscript are done by myself. Mekonnen Tadesse is assisted designing the research and revising the manuscript.

\section{Conflict of Interest Statement}

The authors declare no conflict interests.

\section{Acknowledgements}

I thankful to the EDHS coordinators for their assistant to give data. I also thankful to Mokennon Tadesse (associate professor) for the guidance given during the drafting of the document.

\section{Abbreviations}

CSA: Central Statistical Agency

EDHS: Ethiopia Demographic and Health Survey

U5CM: Under-five child Mortality

UNICEF: United Nations Children Fund

BIC: Bayesian Information Criterion

AIC: Akaike Information Criterion

PRM: Poisson Regression Model

NBRM: Negative Binomial Regression Model

ZIP: Zero Inflated Poisson

ZINB: Zero Inflated Negative Binomial

NB: Negative Binomial

LRT: Likelihood Ratio Test

\section{Appendix}

Table 1. Coding and description of demographic factors.

\begin{tabular}{llll}
\hline No. & variable & levels & Descriptions of variables \\
\hline 1 & MAGEB & $0=<15 \quad 1=15-192=>=20$ & Mother's age at the firth birth \\
2 & CBF & $0=$ No 1 =Yes & Mother's Currently Breastfeeding \\
3 & MS & $0=$ Currently married 1=Currently not married & Mother's Marital status \\
\hline
\end{tabular}

Table 2. Coding and description of socio-economic factors.

\begin{tabular}{|c|c|c|c|}
\hline No. & variable & levels & Descriptions of variables \\
\hline \multirow{4}{*}{4} & \multirow{4}{*}{ MEDU } & $0=$ No education & \multirow{4}{*}{ Mother's level of education } \\
\hline & & $1=$ Primary & \\
\hline & & $2=$ Secondary & \\
\hline & & $3=$ Higher & \\
\hline \multirow{2}{*}{5} & \multirow{2}{*}{ CMW } & $0=\mathrm{No}$ & \multirow{2}{*}{ Mother Currently working } \\
\hline & & $1=$ Yes & \\
\hline \multirow{11}{*}{6} & \multirow{11}{*}{ REG } & $0=$ Tigray & \multirow{11}{*}{ Region } \\
\hline & & $1=$ Affar & \\
\hline & & $2=$ Amhara & \\
\hline & & $3=$ Oromiya & \\
\hline & & 4=Somali & \\
\hline & & 5=Benishangul-Gumuz & \\
\hline & & $6=\mathrm{SNNP}$ & \\
\hline & & 7= Gambela & \\
\hline & & $8=$ Harari & \\
\hline & & 9=Addis Ababa & \\
\hline & & 10=Dire Dawa & \\
\hline \multirow{3}{*}{7} & \multirow{3}{*}{ WI } & $0=$ Poor & \multirow{3}{*}{ Household's wealth index } \\
\hline & & 1=Medium & \\
\hline & & $2=$ Rich & \\
\hline \multirow{2}{*}{8} & \multirow{2}{*}{ PRESD } & $0=$ Urban & \multirow{2}{*}{ Place of residence } \\
\hline & & $1=$ Rural & \\
\hline
\end{tabular}

Table 3. Coding and description environmental factors.

\begin{tabular}{|c|c|c|c|}
\hline No. & variable & levels & Descriptions of variables \\
\hline 9 & SDW & $\begin{array}{l}0=\text { protected } \\
1=\text { unprotected }\end{array}$ & Source of drinking water \\
\hline 10 & $\mathrm{TF}$ & $\begin{array}{l}0=\text { has toilet facility } \\
1=\text { no toilet facility }\end{array}$ & Toilet facility \\
\hline
\end{tabular}


Table 4. Distribution of number of under-5 deaths in urban parts of Ethiopia.

\begin{tabular}{|c|c|c|}
\hline Number of deaths Per mother & Frequency & percent \\
\hline 0 & 1479 & 81.8 \\
\hline 1 & 217 & 12.0 \\
\hline 2 & 65 & 3.6 \\
\hline 3 & 29 & 1.6 \\
\hline 4 & 11 & 0.6 \\
\hline 5 & 3 & 0.2 \\
\hline 6 & 1 & 0.1 \\
\hline Total & 1807 & 100.0 \\
\hline
\end{tabular}

Table 5. Summary data for the number of under-five deaths.

\begin{tabular}{|c|c|c|c|c|c|c|}
\hline & Minimum & Maximum & Mean & Variance & Skewness & kurtosis \\
\hline Number of under- 5 deaths & 0.00 & 7.00 & 0.2839 & 0.541 & 3.658 & 17.723 \\
\hline
\end{tabular}

Table 6. Tests of over-dispersion.

\begin{tabular}{llll}
\hline Statistics & value & Degree of freedom & value \\
\hline Deviance & 1225.381 & 1789 & 0.70 \\
Pearson & 1870.536 & 1789 & 1.05 \\
Chi-Square & 196 freedom & \\
\hline
\end{tabular}

Table 7. Model comparisons.

\begin{tabular}{lll}
\hline Fit statistics & Poisson & NB \\
\hline Log-likelihood & -986.83627 & -970.20254 \\
AIC & 2009.673 & 1978.405 \\
BIC & 2108.662 & 2082.894 \\
\hline
\end{tabular}

Table 8. Model comparisons by Vuong test for non-nested models.

\begin{tabular}{lll}
\hline Model & Vuong statistics(V) & Preferred model \\
\hline ZIP VS Poisson & 5.57 & ZIP \\
ZINB VS NB & 4.54 & ZINB \\
\hline
\end{tabular}

Table 9. Fit statistic for count regression models.

\begin{tabular}{|c|c|c|c|c|}
\hline Criteria & Poisson & NB & ZIP & ZINB \\
\hline $\mathrm{LL}$ & -986.83627 & -970.20254 & -961.6857 & -962.3918 \\
\hline AIC & 2009.673 & 1978.405 & 1964.784 & 1965.371 \\
\hline $\mathrm{BIC}$ & 2108.662 & 2082.894 & 2074.772 & 2080.859 \\
\hline
\end{tabular}

Table 10. Estimated coefficients of ZIP regression model.

\begin{tabular}{|c|c|c|c|c|c|c|}
\hline variables & Coef. & Std. Err. & $\mathbf{z}$ & $\mathbf{P}>|\mathbf{z}|$ & {$[95 \%$} & $\mathbf{C I}]$ \\
\hline \multicolumn{7}{|c|}{ Mother level of education, no education (ref.) } \\
\hline primary & -0.6851459 & 0.118065 & -5.8 & 0.000 & -.916549 & -.4537427 \\
\hline secondary & -1.644393 & 0.284829 & -5.77 & 0.000 & -2.202648 & -1.086139 \\
\hline higher & -1.124482 & 0.3034474 & -3.71 & 0.000 & -1.719228 & -.529736 \\
\hline \multicolumn{7}{|c|}{ Toilet facility, has toilet facility (ref.) } \\
\hline No toilet facility & 0.4553794 & 0.1026778 & 4.44 & 0.000 & .2541347 & .6566242 \\
\hline \multicolumn{7}{|c|}{ Mother age at the first birth, $<15$ (ref.) } \\
\hline 20 and above & -0.6916586 & 0.1660779 & -4.16 & 0.000 & -1.017165 & -.3661518 \\
\hline \multicolumn{7}{|c|}{ Current mother working, no (ref.) } \\
\hline yes & 0.4616567 & 0.0971435 & 4.75 & 0.000 & .271259 & .6520544 \\
\hline cons & -0.6435852 & 0.1602475 & -4.02 & 0.000 & -.9576645 & -.3295059 \\
\hline $\begin{array}{l}\text { log(children ever born) } \\
\text { inflate }\end{array}$ & 1.000 & & & & & \\
\hline \multicolumn{7}{|l|}{$\begin{array}{l}\text { inflate } \\
\text { REG, Tigray (ref.) }\end{array}$} \\
\hline Oromiya & 0.0361875 & 0.6850303 & 0.05 & 0.958 & -1.306447 & 1.378822 \\
\hline Somali & -1.367464 & 0.8548237 & -1.6 & 0.11 & -3.042888 & .3079594 \\
\hline Benshangul- Gumuz & -0.1943995 & 0.7757449 & -0.25 & 0.802 & -1.714832 & 1.326032 \\
\hline
\end{tabular}




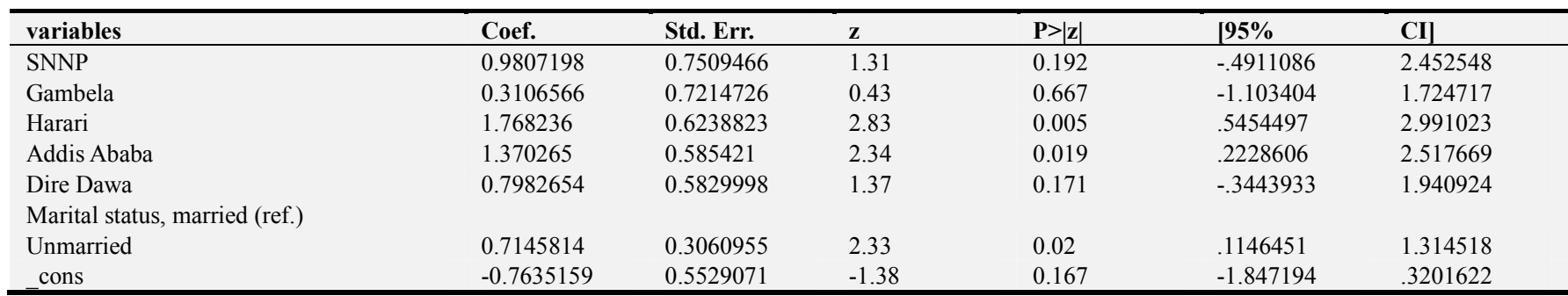

\section{References}

[1] UNICEF. The state of the world's children 2008: Child survival. Unicef; 2007.

[2] Demographic E. Health Survey Central Statistical Agency Addis Ababa. Ethiopia ICF International Calverton, Maryland, USA. 2011: 180-6.

[3] Gideon R., (2000). "Analysis of regional differentials in under-five mortality in Kenya using a count-data regression model", ACAP Working Paper No 14.

[4] Lambert, D., "Zero-inflated Poisson regression, with an application to defects in Manufacturing", Technometrics, 1992; 34: 1-14.

[5] Vuong QH. Likelihood ratio tests for model selection and nonnested hypotheses. Econometrica: Journal of the Econometric Society. 1989 Mar 1: 307-33.

[6] Amare, D., Belaineh, G., and Fasil, T. (2007). Determinants of under-five mortality in Gilgel Gibe Field Research Center, Southwest Ethiopia, Ethiopian journal of health devlopment, vol. 21 (2): 117-124.

[7] Ettarh RR, Kimani J. Determinants of under-five mortality in rural and urban Kenya. Rural \& Remote Health. 2012 Jan 1; 12 (1).

[8] Gideon R., (2012). Under-five mortality differentials in urban East Africa: a study of three capital cities (Nairobi, Dar-esSalaam and Kampala). African Population Studies Vol 26, 1.

[9] Heins F, Tesfaye M, Valente P. Infant and child mortality in urban Ethiopia: with special reference to socio-demographic and housing conditions in urban areas and Addis Ababa. Indepth analysis from the 1994 Population and Housing Census of Ethiopia.

[10] Bariagaber, H. (2013). Housing Correlates of Under-Five 1 Mortality in Urban Ethiopia: A Paper Presented at the XXVII IUSSP International Conference, Busan, Korea, 26-31 August 2013. Department of Population Studies, P/Bag UB 00705.

[11] Tibebu, G. (2011): Identification of risk factors and regional differentials in under-five mortality in Ethiopia using multilevel count model, Addis Ababa University.

[12] Desta, M. (2011). Infant and Child Mortality in Ethiopia: The role of Socioeconomic, Demographic and Biological factors in the previous five years period of 2000 and 2005, Lund University.

[13] Chowdhury, H. A (2013). Determinants of Under-Five Mortality in Bangladesh. Journal of statistics, Vol. 3, pp 213219. Available from http://dx.doi.org/10.4236/ojs.2013.33024. 